\title{
ERRATUM
}

\section{Foramen magnum meningiomas: surgical results and risks predicting poor outcomes based on a modified classification}

TO THE EDITOR: Thank you for publishing our article, "Foramen magnum meningiomas: surgical results and risks predicting poor outcomes based on a modified classification" (J Neurosurg [epub ahead of print May 13, 2016; DOI: 10.3171/2016.2.JNS152873]).

After our article had been published online, we realized that the JNS Publishing Group's style of listing author affiliations does not conform with the requirements of our institution. We apologize for not noticing the difference in styles before publication, but we appreciate the flexibility shown by the JNS Publishing Group in allowing us to relist our authors and affiliations in the following manner:

Da Li, MD, ${ }^{1-4}$ Zhen $\mathrm{Wu}, \mathrm{MD}, \mathrm{PhD},{ }^{1-4}$ Cong Ren, $\mathrm{MD},{ }^{1-4}$ Shu-Yu Hao, MD, PhD, ${ }^{1}$ Liang Wang, $\mathrm{MD}, \mathrm{PhD},{ }^{1} \mathrm{Xin}-\mathrm{Ru}$ Xiao, MD, PhD,${ }^{1}$ Jie Tang, $\mathrm{MD}, \mathrm{PhD},{ }^{1}$ Yong-Gang Wang, $\mathrm{MD}, \mathrm{PhD},{ }^{1}$ GuoLu Meng, MD, PhD, ${ }^{1}$ Li-Wei Zhang, MD, PhD, ${ }^{1-4}$ and Jun-Ting Zhang, $\mathrm{MD}^{1-4}$

${ }^{1}$ Department of Neurosurgery, Beijing Tiantan Hospital, Capital Medical University, Beijing, People's Republic of China; ${ }^{2}$ China National Clinical Research Center for Neurological Diseases; ${ }^{3}$ Center of Brain Tumor, Beijing Institute for Brain Disorders; and ${ }^{4}$ Beijing Key Laboratory of Brain Tumor, Beijing Tiantan Hospital, Capital Medical University, Beijing, People's Republic of China

The authors' affiliation list has been corrected online as of October 21,2016, and is correct in this issue.

Jun-Ting Zhang, MD

Beijing Tiantan Hospital, Capital Medical University, Beijing, People's Republic of China 